\title{
Cuentas conjuntas o separadas: Administración del dinero en familias de primera unión y reconstituidas
}

\section{Joint or separate accounts: Money management on first union and reconstructed families}

\author{
Karen Ripoll-Núñez $\quad$ Karen Martínez Arrieta² \\ Universidad de los Andes, Departamento de Psicología, Bogotá, Colombia
}

(Rec: septiembre de 2012 - Acep: octubre de 2012)

\begin{abstract}
Resumen
Este estudio se propuso explorar los sistemas de administración del dinero empleados por parejas de primera unión y reconstituidas. Son 26 parejas heterosexuales (12 parejas de primera unión y 14 reconstituidas) las que respondieron a una entrevista en profundidad sobre la manera cómo aportan económicamente al presupuesto familiar, la administración de esos aportes, y su percepción sobre qué tan justas son las decisiones sobre el dinero en su relación. El análisis de las temáticas que emergieron permitió identificar cinco sistemas de administración: salario completo, mesada, fondo común, manejo independiente y manejo independiente con fondo común. Contrario a lo señalado en otros estudios, las parejas de primera unión empleaban sistemas de manejo independiente o manejo independiente con fondo común. Sin embargo, en parejas reconstituidas se encontró principalmente el sistema de fondo común. Los resultados se discuten en relación con la literatura acerca de la administración del dinero en diferentes tipos de uniones maritales.

Palabras clave: decisiones, administración del dinero, parejas, primera unión y reconstituidas.
\end{abstract}

\begin{abstract}
The purpose of this study was to explore the finances management systems employed by first union and remarried couples. 26 heterosexual couples (12 first union and 14 remarried couples) answered to an in-depth interview about the ways each partner contributes to the family budget, the ways they administer money, and the extent to which decisions regarding money in their relationship are fair. Emerging themes allowed us to identify five management systems: whole salary, allowance, shared account, independent management, and independent management with shared account. In contrast with existing evidence, first union couples employed more independent management or independent management with shared account systems. However, remarried couples employed primarily a shared account system. Results are discussed in relation to existing literature regarding finance management systems in different types of marital unions.
\end{abstract}

Keywords: decisions, money management, couples, first union and remarried.

Correspondencia a: Carrera 1 Este 18 A 10, Bogotá, Colombia. Tel: (571) 339-4949. Ext. 2579. E-mail: kripoll@uniandes.edu.co.

MSc. Psicóloga, Práctica independiente. E-mail: ktmartineza@yahoo.com. 


\section{Introducción}

La integración de recursos económicos, tanto a nivel práctico como legal, ha sido un aspecto central de la unión matrimonial en diversas culturas (Lauer $\&$ Yodanis, 2007). Sin embargo, varios autores han llamado la atención sobre una tendencia hacia la individualización del matrimonio en occidente (Amato, Booth, Johnson \& Rogers, 2007; Cherlin, 2004). En esta tendencia, el fundamento económico del matrimonio pasa a un segundo plano y el interés central que fundamenta la unión es el amor y la intimidad emocional. Los esposos mantienen sus identidades como personas independientes y esperan que cada uno pueda proveer económicamente por sí mismo (Lauer \& Yodanis, 2011).

En las últimas décadas se han observado cambios significativos en Colombia en relación con los logros educativos y laborales de las mujeres, que hacen pensar en una mayor independencia económica de las mismas en relación con sus parejas. Datos recientes sobre mujeres en edad fértil en Colombia, revelan que aproximadamente el 53\% de las que están en unión marital trabajan (Profamilia, 2010). Empero, las probabilidades de participación laboral de las mujeres en unión marital con hijos disminuyen cuando cuentan con el apoyo económico de su compañero (Alvis-Guzmán, YánezContreras, Quejada-Pérez, Acevedo-González \& Del Río-Carrasquilla, 2010). Por otra parte, a pesar de que en promedio las mujeres tienen $6 \%$ más años de educación que los hombres en el grupo de asalariados, los salarios promedio de las mujeres en el mercado laboral urbano son $10 \%$ menores a los de los hombres (Galvis, 2010). Es así como la maternidad y la desigualdad en los salarios, entre otros factores, pueden influir en la participación de las mujeres en decisiones relacionadas con el dinero al interior de la pareja.

Aunque existen estudios demográficos en Colombia sobre la participación de la mujer en las decisiones sobre dinero en la familia (Profamilia, 2010), no se encuentran estudios que exploren directamente los sistemas que establecen las parejas para administrar sus recursos económicos en su relación marital. ¿Existe una tendencia en las parejas de nuestro medio a integrar los ingresos en un fondo común o, por el contrario, prefieren los cónyuges administrar sus ingresos de manera independiente? ¿Quién tiene el control sobre las decisiones relacionadas con la administración del dinero?

Por otra parte, diversos estudios en otros países han planteado que las personas -especialmente las mujeres- que vuelven a formar uniones maritales después de divorciarse tienen expectativas diferentes en relación con la equidad y la distribución del poder en decisiones relacionadas con el dinero, las tareas domésticas y la crianza de los hijos (Ganong \& Coleman, 2004). Asimismo, se ha encontrado que al formar una familia reconstituida, las parejas adoptan sistemas de administración del dinero que se adecuan a sus necesidades particulares (por ejemplo, mantener a hijos de uniones anteriores) (Burgoyne \& Morison, 1997). Sin embargo, pocos estudios han explorado qué diferencias se encuentran en los sistemas empleados por parejas en familias de primera unión y reconstituidas en Colombia para administrar sus ingresos.

\section{Dinero en el matrimonio}

La manera como las parejas manejan los recursos económicos nos proporciona pistas sobre aspectos clave de la dinámica relacional en las parejas. Por ejemplo, la decisión de la pareja de tener una cuenta compartida para gastos o mantener cuentas separadas puede indicarnos el nivel de compromiso e integración en la relación (Heimdal \& Houseknecht, 2003; Oropesa, Landale \& Kenkre, 2003; Treas, 1993), así como también puede ser un indicativo del nivel de igualdad entre los cónyuges (Blumstein \& Schwartz, 1991).

Pahl (1989) desarrolló la siguiente tipología en relación con la administración del dinero en parejas heterosexuales, en la cual se tiene en cuenta no sólo la manera como cada individuo aporta al presupuesto familiar, sino quién tiene la responsabilidad principal de administrar y decidir sobre el dinero (control del dinero): 1) Sistema del salario completo: existe un proveedor económico principal (generalmente el hombre) que da la mayor parte de sus ingresos para el sostenimiento del hogar, pero retiene algo de dinero para sus gastos personales. La pareja (generalmente la mujer) usa los aportes del proveedor principal y sus propios ingresos para cubrir los gastos. 2) Sistema de mesada: el proveedor principal (generalmente el hombre) da una mesada a su pareja para los gastos de mantenimiento del hogar y retiene una porción de su salario para gastos personales. 3) Sistema de fondo común o compartido: todos o casi todos los ingresos se tratan como un recurso colectivo. Los dos miembros de la pareja administran el fondo y toman parte en las decisiones. 4) Sistema de manejo independiente: cada individuo controla sus propios ingresos y asume responsabilidad por sus gastos personales y una parte de los gastos familiares. Ninguno de los dos tiene control sobre todos los ingresos del hogar. Es posible que 
existan arreglos mixtos dentro de este sistema, en los que hay una cuenta común para gastos familiares a la que aportan las dos personas.

Los estudios indican que en diversas sociedades el sistema más común de administración del dinero es el denominado fondo común (Heimdal \& Houseknecht, 2003; Pahl, 2008; Vogler, 2005). La mayoría de las parejas adoptan con el tiempo este sistema, aunque al inicio de su vida conyugal cada individuo haya manejado de manera independiente su dinero (Burgoyne, Reibstein, Edmunds \& Dolman, 2007). A pesar de estas tendencias, en los últimos años se ha incrementado el número de parejas que adoptan un sistema de manejo independiente (o cuentas separadas) (Vogler, Brockmann \& Wiggins, 2006). Estas parejas manifiestan que tener cuentas separadas les ayuda a mantener independencia en su relación (Burgoyne, Reibstein, Edmunds \& Routh, 2010).

\section{Inequidad y manejo del dinero}

Durante siglos, el propósito central del matrimonio como institución social fue preservar los intereses económicos de las familias de los contrayentes (Coontz, 2005; Mintz \& Kellogg, 1988). Este matrimonio patriarcal se caracterizó por el control masculino del dinero. A medida que la institución matrimonial ha cambiado a una unión basada en la compañía y apoyo mutuo entre los cónyuges, el fondo común o cuenta conjunta se convierte en el sistema predominante para el manejo de los recursos (Zelizer, 1989). Sin embargo, ¿qué tan equitativas son las prácticas de administración del dinero en las parejas?

Aunque tanto hombres como mujeres inician el matrimonio con la expectativa de establecer condiciones de igualdad en asuntos de dinero (Burgoyne \& Routh, 2001), esto no siempre se hace realidad en la práctica (Burgoyne, 2004; Webley et al., 2001). Estudios empíricos sugieren que diferentes factores, tanto a nivel individual como contextual, se asocian a los sistemas de manejo del dinero que desarrollan las parejas.

\section{Factores Individuales}

En la investigación empírica se han identificado cuatro variables de los miembros de la pareja que influyen en la manera como se administra el dinero. Una de ellas, tal vez la principal, tiene que ver con el origen de los ingresos en el hogar o la contribución relativa que hacen los miembros de la pareja al sustento económico de la familia. De acuerdo con la teoría de los recursos (Blood \& Wolfe, 1960), quien aporta más ingresos a la familia probablemente mantendrá más control sobre esos recursos; en tanto que las relaciones serán más equitativas en la medida en que los esposos contribuyan por igual al sustento familiar. Aun cuando los miembros de la pareja perciban los ingresos como un bien común, ambos son conscientes de quién aporta y cuánto aporta cada uno. Esto parece cierto aun en casos en los que la pareja tiene una cuenta compartida para cubrir los gastos familiares (Burgoyne, 1990, Burgoyne \& Lewis, 1994).

El segundo factor que influye en el manejo del dinero es el nivel de ingresos familiar. Se han hallado diferencias en los sistemas de administración que adoptan las parejas de acuerdo con el estrato socioeconómico. Empero, cuando los hombres son los principales proveedores tienen más probabilidad de ejercer control sobre el dinero independientemente del sistema de administración empleado por la pareja (Pahl, 1989; Wilson, 1987).

Las expectativas y creencias sobre el matrimonio -por ejemplo, creencias sobre roles de género- también inciden en la administración del dinero. En parejas con visiones más tradicionales se encuentra mayor control de lo económico por parte del hombre, en tanto que en parejas con visiones menos tradicionales se espera una participación más equitativa de ambos miembros de la pareja (Pahl, 1989). Algunos autores afirman que estas creencias constituyen reglas de conducta institucionalizadas (Martin, 2004).

Por último, las características de la relación y las experiencias que anteceden a la misma pueden influir también en el sistema de administración del dinero. De acuerdo con Treas (1993), las parejas establecen sistemas para manejar el dinero que minimizan los costos de sus decisiones. Cuando se percibe que el matrimonio es inestable, las parejas adoptan sistemas de administración en los que sea más fácil separar sus recursos económicos en caso de una eventual separación (Burgoyne \& Morrison, 1997). Asimismo, las parejas en las que uno o ambos miembros tuvieron una relación anterior que terminó en divorcio, tienden a mantener cuentas separadas (Burgoyne \& Morison, 1997; Heimdal \& Houseknecht, 2003).

\section{Factores contextuales}

Otros autores han señalado que es necesario considerar el papel de factores contextuales en el tipo de sistemas de administración del dinero que implementan las parejas (Lauer \& Yodanis, 2011; Yodanis \& Lauer, 
2007). Específicamente, los sistemas que implementan las parejas están inmersos en los valores culturales e ideologías sociales (Treas, 1993) y se ven moldeados por las instituciones sociales (Brinton \& Nee, 1998).

Entre estas creencias y prácticas culturales se encuentra la definición institucional del proveedor (Tichenor, 1999; Vogler \& Pahl, 1993), que prima en cada sociedad. Esta se refiere a un conjunto de creencias dominantes acerca de los roles aceptables para hombres y mujeres en el matrimonio y la expectativa acerca de quién debe ser el principal proveedor económico (Blumstein \& Schwartz, 1985). Quien se define institucionalmente como el proveedor tiene más poder en las decisiones sobre el manejo del dinero. Yodanis y Lauer (2007) encontraron en 21 países que la presencia de una definición institucional en la que se considerara a ambos miembros de la pareja como proveedores económicos tenía mayor efecto en la actitud de las parejas hacia compartir la administración del dinero, que los ingresos reales aportados por cada miembro de la pareja a la economía familiar.

\section{Administración del dinero en familias reconstituidas}

Estudios empíricos han demostrado que las mujeres perciben una mayor participación en decisiones sobre la administración del dinero en su nueva unión marital, cuando la comparan con sus uniones anteriores (Burgoyne \& Morison, 1997; Pasley, Sandras \& Edmondson, 1994). Sin embargo, esto no necesariamente significa que las decisiones sobre el dinero se compartan de manera equitativa entre los miembros de la pareja (Ganong \& Coleman, 2004).

Se han descubierto al menos cuatro formas en que las parejas administran el dinero en familias reconstituidas: a) fondo común (Fishman, 1983; Coleman \& Ganong, 1989); b) cuentas separadas o manejo independiente (Fishman, 1983); c) arreglo "mixto" (manejo independiente con fondo común) (Pasley et al., 1994); y, d) mesada.

Existe poca literatura sobre la administración del dinero en familias reconstituidas de países latinoamericanos $\mathrm{y}$, específicamente, no hay estudios comparativos que examinen los sistemas de administración y el control del dinero en parejas de primera unión y reconstituidas. Este estudio se propuso responder a las siguientes preguntas de investigación: a) ¿qué caracteriza las experiencias de parejas de primera unión y reconstituidas en relación con la administración del dinero?; b) ¿qué sistemas emplean las parejas para el manejo de sus ingresos (fondo común, cuentas separadas, arreglos mixtos)?; c) ¿quién (es) tiene el control principal en la administración del dinero?; y, d) ¿cuál es la percepción de las parejas sobre la "justicia" en los sistemas de administración y control del dinero que adoptan cotidianamente en sus familias?

\section{Método}

\section{Participantes}

Los participantes fueron 26 parejas heterosexuales: 12 en su primera unión y 14 en su segunda o tercera unión (familias reconstituidas). De las doce parejas que se encontraban en su primera relación matrimonial, 8 estaban unidas en matrimonio y 4 parejas cohabitaban. La duración promedio de la relación en estas parejas era de 13 años ( $D E=11,07$ años). La edad de los hombres oscilaba entre 24 y 68 años $(M=37,67, D E=11,85)$ y la de las mujeres entre 24 y 64 años $(M=36,33$, $D E=11,28)$. El 49 por ciento de los hombres tenían estudios universitarios y el $74 \%$ eran empleados. El cincuenta por ciento de las mujeres tenían título universitario y eran empleadas de tiempo completo. Se halló una diferencia del cuarenta y cinco por ciento entre el ingreso promedio de las mujeres ( $\$ 2.633 .333$ pesos) y el de los hombres ( $\$ 4.814 .166$ pesos) en las parejas de primera unión.

En las 14 parejas de familias reconstituidas, uno o los dos miembros de la pareja había tenido una (o más) relación de convivencia (matrimonio o cohabitación) previa a la relación actual y tenía hijos producto de esa relación anterior. Tres de las 14 parejas eran casadas y 11 cohabitaban. La duración promedio de la relación de pareja actual de los participantes era de 6,03 años ( $D E=4,38$ años). La edad de los hombres oscilaba entre 26 y 65 años $(M=44,43, D E=12,81)$. La edad promedio de las mujeres era de 39,43 años $(D E=9,34)$ y oscilaba entre 24 y 58 años. Sesenta y uno por ciento de los hombres trabajaba tiempo completo y cincuenta por ciento tenía estudios universitarios. El 43 por ciento de las mujeres tenía un trabajo de tiempo completo y el $57 \%$ había cursado estudios universitarios. Se encontró una diferencia del treinta y cinco por ciento entre el ingreso promedio de las mujeres ( $\$ 2.305 .714$ pesos) y el de los hombres (\$3.557.142 pesos) en la muestra de parejas reconstituidas. 
Al comparar los hombres y mujeres de las parejas de primera unión y las reconstituidas, no hubo diferencias significativas en términos de edad $(t=-, 77, n s ; t=$ $-1,38, n s$, para hombres y mujeres, respectivamente) e ingreso salarial ( $t=, 28 n s ; t=, 64, n s$, para hombres y mujeres, respectivamente). Sin embargo, se encontró una diferencia estadísticamente significativa en el tiempo de convivencia, que indica que las parejas de primera unión habían estado unidas más tiempo que las parejas reconstituidas $(t=2,17, p<0,05)$.

\section{Instrumentos}

Se diseñó una entrevista semi-estructurada que exploraba: a) datos demográficos generales del participante; b) contribución de cada miembro de la pareja a los ingresos familiares; c) acuerdos establecidos para la administración del dinero (quién administra el dinero, para qué tipo de gastos); d) participación de los miembros de la pareja en decisiones sobre dinero; $y, e)$ evaluación personal sobre justicia en la administración del dinero en la relación. Los entrevistadores usaron la guía de entrevista de manera flexible e indagaron sobre detalles y ejemplos específicos para obtener descripciones de las experiencias de los participantes.

\section{Procedimiento}

Las parejas participantes fueron contactadas a través de conocidos y empleando una estrategia de bola de nieve. Para participar en el estudio, las parejas de primera unión debían reunir las siguientes características: a) la unión marital actual debería ser la primera para ambas personas; b) los miembros de la pareja no habían convivido o tenido hijos con parejas anteriores a su unión actual; c) la pareja tenía al menos un hijo producto de la relación actual; y d) la pareja tenía más de un año de estar conviviendo. En el caso de las familias reconstituidas, la pareja debía cumplir con dos requisitos: a) un cónyuge (o ambos) tenía al menos un hijo producto de una relación de convivencia anterior; y, b) la pareja tenía más de un año de estar conviviendo. Dos miembros del equipo investigador entrevistaban por separado a los integrantes de la pareja. Antes de iniciar la entrevista, cada participante leía un formato de consentimiento en el que se explicaban los objetivos del estudio y los procedimientos que se seguirían para proteger su identidad y la confidencialidad de los datos. Las entrevistas tenían una duración promedio de 75 minutos y fueron grabadas en formato de audio. Posteriormente, las entrevistas fueron transcritas textualmente para facilitar su análisis. En las transcripciones se reemplazaron los nombres de los participantes y sus hijos por seudónimos.

\section{Análisis de datos}

Se empleó el método de comparación constante (Strauss, 1987). Este proceso consiste en la codificación y análisis simultáneo de los datos (Willig, 2001). Los datos fueron examinados repetidas veces para identificar similitudes y diferencias. A partir de este análisis se construyó un árbol de categorías y se realizó la codificación de las transcripciones empleando el programa para análisis cualitativo de datos ATLAS.ti 5 (Muhr, 2004). Posteriormente, se identificaron temáticas a partir de las respuestas codificadas y se elaboraron memos interpretativos (Strauss \& Corbin, 1990).

\section{Resultado}

A partir del análisis de las transcripciones, se construyó un árbol de categorías (ver Figura 1). Teniendo en cuenta los tres principales focos de la entrevista (aporte económico, manejo del dinero y percepción de justicia), se obtuvieron categorías tras las respuestas de hombres y mujeres. Así, sobre el aporte económico emergieron dos categorías centrales (origen y proporción) y sus respectivas sub-categorías. Con relación al manejo del dinero, se identificaron dos categorías: sistema de administración y control de decisiones. Finalmente, las percepciones de los participantes sobre el nivel de justicia en la administración del dinero se dividieron en dos categorías principales (sistema justo y sistema injusto).

Una vez codificadas las entrevistas, se utilizó la tipología propuesta por Pahl (1989) para tipificar el sistema de administración del dinero empleado por cada pareja. Para ello se identificaron las temáticas presentes en la descripción de los miembros de la pareja sobre: quién(es) aportaban dinero al sustento familiar, cómo se usaban y administraban esos ingresos y quién(es) tenían mayor responsabilidad en la administración de los ingresos. A continuación se describe cada uno de los sistemas de administración del dinero identificados y se presentan ejemplos de cada uno. Los fragmentos textuales de las transcripciones se inician con el seudónimo del participante, edad y el tipo de familia (primera unión o reconstituida). 
Figura 1.

Árbol de categorías.

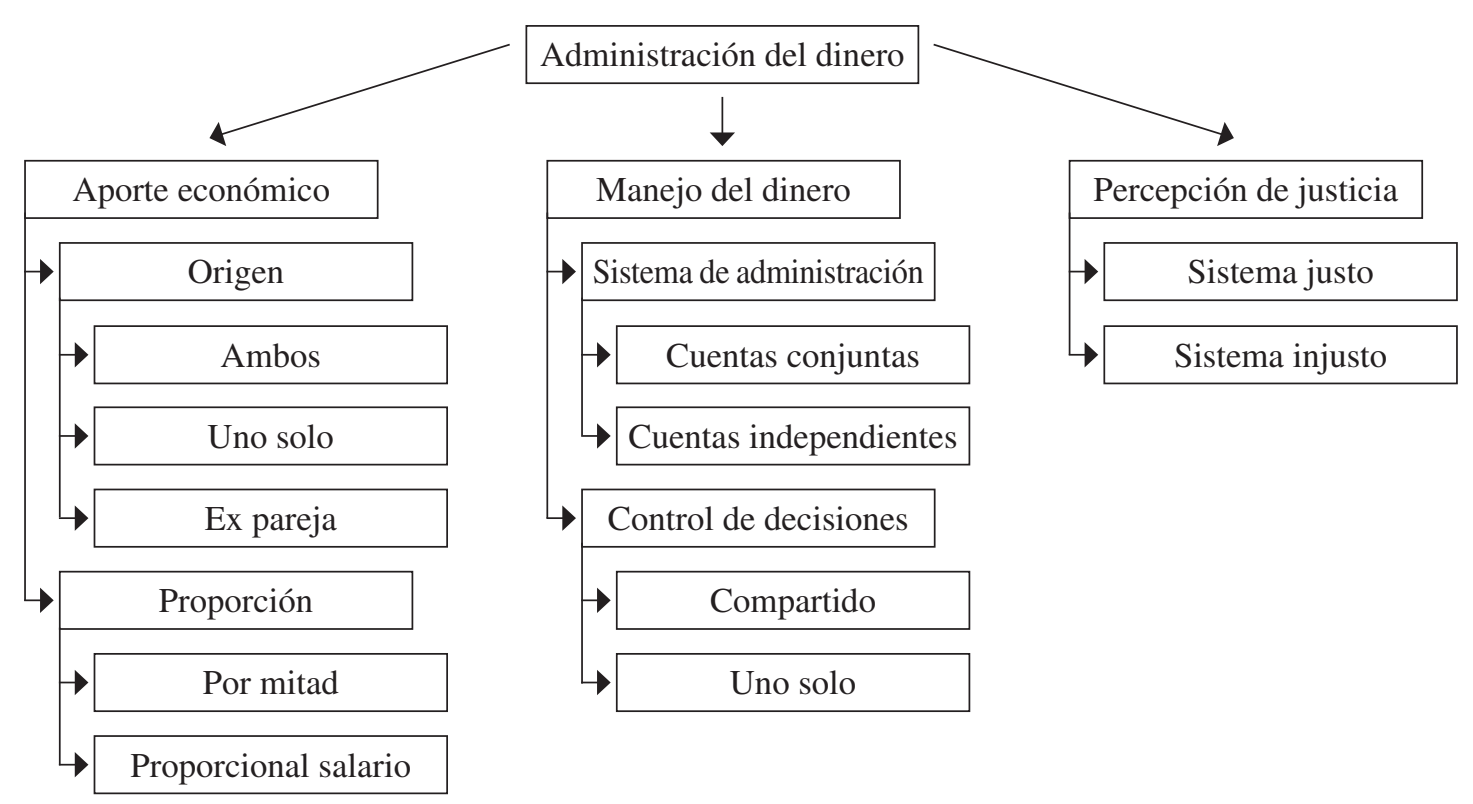

\section{Sistemas de administración}

Como se aprecia en la Tabla 1, en todas las parejas de primera unión el hombre tenía ingresos superiores a la mujer. En algunos casos (4 parejas), la diferencia de salario entre hombres y mujeres era superior al $50 \%$. A pesar de esta diferencia en ingresos, estas parejas no señalaron al hombre como el principal proveedor económico en sus familias. Esto sólo sucedió en las cuatro parejas donde la mujer no tenía ingresos. En las familias reconstituidas existían diferentes situaciones en relación con los ingresos que aportaban hombres y mujeres. En el 50\% de estas parejas (7 parejas), el hombre tenía ingresos que superaban en 50\% o más los ingresos de su esposa. Por otra parte, en cuatro hogares los ingresos de la mujer eran superiores a los del hombre, aunque en sólo 2 parejas el salario de ella superaba en más del 50\% el ingreso de él. Sólo en una de las parejas, la mujer no devengaba salario.

En la clasificación de las parejas en la tipología que se presenta a continuación se tuvo en cuenta no sólo a quiénes aportaban al presupuesto y los acuerdos para administrar esos ingresos, sino también a quién(es) tenía(n) mayor control sobre el dinero. Como puede apreciarse en la Tabla 1, aunque dos parejas tengan sistemas similares de administración, el control del dinero puede estar en manos de uno o ambos miembros de la pareja.

Salario completo. Las parejas que empleaban este sistema se caracterizaban por tener un proveedor económico principal, que aportaba la mayor parte de su salario a los gastos familiares y retenía un porcentaje para gastos personales. Ocasionalmente, los ingresos del otro miembro de la pareja también se empleaban para cubrir gastos.

Leonardo (58 años, reconstituida): "Claro, el mayor porcentaje lo aporto yo pues por los ingresos...ella también tiene sus negocitos particulares y también aporta, muchas veces para pagar algún recibo o alguna cosa para la casa. Pero la mayor cantidad la aporto yo".

Seis parejas reconstituidas empleaban este sistema (23\% de la muestra). En cuatro de ellas, el principal proveedor era el hombre. Sin embargo, no se encontró que la mujer tuviese a cargo el control del dinero en esas parejas, como lo describe Pahl (1989) en su estudio. De hecho, en dos parejas las dos personas administran el dinero conjuntamente (Leonardo y Laura; Omar y Olga) y en otras dos era el hombre quien administra el dinero (Duván y Diana; Julián y Juliana). Cuando 
Tabla 1.

Conformación de parejas participantes, ingresos y sistemas de administración del dinero.

\begin{tabular}{|c|c|c|c|c|c|c|c|c|c|}
\hline $\begin{array}{c}\text { Pareja } \\
\text { (seudónimos) }\end{array}$ & $\begin{array}{l}\text { Edad } \\
\text { (años) }\end{array}$ & $\begin{array}{c}\text { Tipo } \\
\text { Familia }\end{array}$ & $\begin{array}{l}\text { Estatus } \\
\text { Unión } \\
\text { (años) }\end{array}$ & $\begin{array}{c}\text { Hijos } \\
\text { Unión } \\
\text { Actual } \\
\end{array}$ & $\begin{array}{c}\text { Hijos } \\
\text { Uniones } \\
\text { Anteriores } \\
\end{array}$ & $\begin{array}{l}\text { Ingresos } \\
\text { (pesos) }\end{array}$ & $\begin{array}{c}\text { Diferencia } \\
\text { Salarial } \\
\text { (porcentaje) } \\
\end{array}$ & Sistema Administración & $\begin{array}{l}\text { Control } \\
\text { Dinero }\end{array}$ \\
\hline Andrés & 45 & $\mathrm{P}$ & Casados & 2 & 0 & 4.000 .000 & 13 & $\begin{array}{l}\text { Independiente con fondo } \\
\text { común }\end{array}$ & Compartido \\
\hline Amalia & 43 & & (20) & & 0 & 3.500 .000 & & & \\
\hline Bernardo & 33 & $\mathrm{P}$ & Casados & 1 & 0 & 6.000 .000 & 17 & Independiente & Compartido \\
\hline Beatriz & 35 & & (5) & & 0 & 5.000 .000 & & & \\
\hline Carlos & 42 & $\mathrm{P}$ & Casados & 2 & 0 & 1.500 .000 & 67 & Independiente & Hombre \\
\hline Camila & 42 & & (20) & & 0 & 500.000 & & & \\
\hline David & 68 & $\mathrm{P}$ & Casados & 3 & 0 & 400.000 & 100 & Mesada & Mujer \\
\hline Daniela & 64 & & $(40)$ & & 0 & 0 & & & \\
\hline Enrique & 31 & $\mathrm{P}$ & Unión libre & 1 & 0 & 1.500 .000 & 53 & $\begin{array}{l}\text { Independiente con fondo } \\
\text { común }\end{array}$ & Compartido \\
\hline Esther & 33 & & (12) & & 0 & 700.000 & & & \\
\hline Francisco & 46 & $\mathrm{P}$ & Casados & 4 & 0 & 3.000 .000 & 100 & Mesada & Compartido \\
\hline Fernanda & 43 & & $(23)$ & & 0 & 0 & & & \\
\hline Gabriel & 34 & $\mathrm{P}$ & Casados & 1 & 0 & 23.000 .000 & 57 & $\begin{array}{l}\text { Independiente con fondo } \\
\text { común }\end{array}$ & Hombre \\
\hline Gloria & 34 & & (8) & & 0 & 10.000 .000 & & & \\
\hline Humberto & 40 & $\mathrm{P}$ & Casados & 2 & 0 & 12.000 .000 & 17 & Independiente & Compartido \\
\hline Helena & 39 & & $(15)$ & & 0 & 10.000 .000 & & & \\
\hline Ignacio & 24 & $\mathrm{P}$ & Unión libre & 1 & 0 & 2.500 .000 & 52 & Independiente & Compartido \\
\hline Isabel & 24 & & $(2)$ & & 0 & 1.200 .000 & & & \\
\hline Joaquín & 33 & $\mathrm{P}$ & Unión libre & 1 & 0 & 1.000 .000 & 30 & Independiente & Compartido \\
\hline Juana & 26 & & (4) & & 0 & 700.000 & & & \\
\hline Luis & 29 & $\mathrm{P}$ & Unión libre & 1 & 0 & 1.500 .000 & 100 & Mesada & Compartido \\
\hline Liliana & 25 & & (6) & & 0 & 0 & & & \\
\hline Mario & 27 & $\mathrm{P}$ & Casados & 1 & 0 & 1.370 .000 & 100 & Mesada & Compartido \\
\hline Mariana & 27 & & (3) & & 0 & 0 & & & \\
\hline Alfonso & 39 & $\mathrm{R}$ & Unión libre & 0 & 3 & 3.500 .000 & 0 & Fondo común & Compartido \\
\hline Andrea & 38 & & (1) & & 3 & 3.500 .000 & & & \\
\hline César & 36 & $\mathrm{R}$ & Unión libre & & 2 & 12.000 .000 & 75 & Mesada & Hombre \\
\hline Carolina & 28 & & $(4,5)$ & & 0 & 3.000 .000 & & & \\
\hline Duván & 27 & $\mathrm{R}$ & Unión libre & 1 & 0 & 900.000 & 67 & Salario completo & Hombre \\
\hline Diana & 42 & & (5) & & 3 & 300.000 & & & \\
\hline Elkin & 46 & $\mathrm{R}$ & Unión libre & 2 & 2 & 2.500 .000 & 100 & Mesada & Hombre \\
\hline Eliana & 24 & & $(5)$ & & 0 & 0 & & & \\
\hline Fabián & 49 & $\mathrm{R}$ & Unión libre & 1 & 0 & 3.000 .000 & 54 & Fondo común & Compartido \\
\hline Fabiana & 45 & & (8) & & 2 & 6.500 .000 & & & \\
\hline Julián & 32 & $\mathrm{R}$ & Unión libre & 1 & 1 & 3.000 .000 & 67 & Salario completo & Hombre \\
\hline Juliana & 31 & & (9) & & 0 & 1.000 .000 & & & \\
\hline Leonardo & 58 & $\mathrm{R}$ & Unión libre & & 4 & 3.500 .000 & 77 & Salario completo & Compartido \\
\hline Laura & 38 & & $(5)$ & & 3 & 800.000 & & & \\
\hline Miguel & 26 & $\mathrm{R}$ & Unión libre & 1 & 0 & 800.000 & 38 & Fondo común & Mujer \\
\hline María & 25 & & (5) & & 1 & 1.300 .000 & & & \\
\hline Nicolás & 34 & $\mathrm{R}$ & Unión libre & 0 & 0 & 2.800 .000 & 86 & Fondo común & Compartido \\
\hline Nidia & 33 & & $(0,5)$ & & 1 & 400.000 & & & \\
\hline Omar & 64 & $\mathrm{R}$ & Casados & 1 & 4 & 2.500 .000 & 61 & Salario completo & Compartido \\
\hline Olga & 35 & & $(9,5)$ & & 0 & 980.000 & & & \\
\hline Pablo & 48 & $\mathrm{R}$ & Unión libre & 0 & 3 & 8.000 .000 & 75 & Fondo común & Compartido \\
\hline Paula & 51 & & (6) & & 0 & 2.000 .000 & & & \\
\hline Ricardo & 52 & $\mathrm{R}$ & Unión libre & 0 & 2 & 300.000 & 91 & Salario completo & Compartido \\
\hline Rita & 51 & & (6) & & 2 & 3.500 .000 & & & \\
\hline Santiago & 45 & $\mathrm{R}$ & Casados & 1 & 0 & 4.000 .000 & 0 & Fondo común & Compartido \\
\hline Sonia & 50 & & $(18)$ & & 1 & 4.000 .000 & & & \\
\hline Tito & 66 & $\mathrm{R}$ & Casados & 0 & 1 & 3.000 .000 & 40 & Salario completo & Compartido \\
\hline Tatiana & 51 & & $(1,5)$ & & 1 & 5.000 .000 & & & \\
\hline
\end{tabular}


la mujer era la proveedora principal (Ricardo y Rita; Tito y Tatiana), el control del dinero era compartido por los dos miembros de la pareja. No había parejas de primera unión que emplearan este sistema, en la muestra del estudio.

Mesada. En las parejas que utilizaban este sistema (23\% de la muestra: 4 primera unión; 2 reconstituidas), se reconocía a un proveedor principal que asignaba una parte de sus ingresos para los gastos de la familia. La mujer no devengaba salario en todas las parejas de primera unión y en una de las parejas reconstituidas que empleaban este sistema.

David (68 años, primera unión): "A mí es al que le toca conseguir para todos los servicios, lo que yo consigo, yo se lo paso a ella y ella dispone, pero como ella no trabaja, entonces yo le doy a ella lo de las necesidades diarias".

Sólo en una pareja reconstituida la mujer recibía ingresos, pero los destinaba a sus gastos personales porque del salario de su esposo salía la mesada para los gastos de la familia (César y Carolina). Existían diferentes situaciones en relación con el control del dinero en las parejas que utilizaban este sistema; en tres parejas de primera unión la mesada se administraba de manera compartida. En tanto que en las dos parejas reconstituidas, el hombre tenía control sobre el dinero de la mesada. Sólo se encontró la situación típica de las parejas que emplean el sistema de mesada -la mujer administra la mesada asignada por el hombre (Pahl, 1989)-, en una pareja de primera unión (David y Daniela).

Fondo común. En este sistema, la totalidad de los ingresos de ambos miembros de la pareja se juntan en una cuenta para cubrir los gastos de la familia. La pareja trata todos los ingresos como un recurso colectivo.

Pablo (48 años, pareja reconstituida): "Hacemos un solo fondo, una sola cuenta...con lo que haya, de la tarjeta que haya plata se paga".

Este sistema se encontró únicamente en seis parejas reconstituidas (23\% de la muestra). Cinco de estas parejas señalaron que el dinero del fondo común era administrado de manera compartida por los miembros de la pareja. En dos de estas parejas, los ingresos del hombre y la mujer eran iguales (Alfonso y Andrea; Santiago y Sonia), en tanto que en las otras dos los hombres tenían un ingreso superior (Nicolás y Nidia; Pablo y Paula). Sólo en dos de las parejas que empleaban el sistema de fondo común, la mujer tenía ingresos superiores al hombre. Sin embargo, en tanto que una de estas parejas administraba el dinero de manera compartida (Fabián y Fabiana), en la otra pareja la mujer tenía el control sobre la administración de los gastos (Miguel y María). De las 6 parejas que empleaban este sistema, cinco convivían en unión libre. Dos mujeres (Fabiana y Nidia) reportaron que lo que recibían de sus ex esposos para el sostenimiento de sus hijos iba también al fondo común para gastos familiares. Este dinero lo utilizaban para pagar algún servicio o para gastos escolares de los hijos.

Manejo independiente. En este sistema, los dos miembros de la pareja aportan una porción de sus ingresos al sostenimiento del hogar y destinan el resto a sus gastos personales. No obstante, los aportes de cada miembro de la pareja no van a una cuenta común, sino cada uno maneja su propia cuenta. Las cinco parejas de la muestra que se caracterizaban por este sistema eran de primera unión (19\% de la muestra). En todas estas parejas, aunque existía una diferencia en ingresos -el salario del hombre era superior-, cada persona aportaba al presupuesto familiar en proporción a su salario.

Bernardo (33 años, primera unión): "Es proporcional a lo que ganamos. Nosotros tomamos los ingresos y sumamos los gastos, y proporcionalmente a lo que cada uno recibe es lo que cada uno gasta".

Cuatro de estas parejas administraban el dinero para gastos familiares y tomaban decisiones de manera conjunta. Sin embargo, en una de las cinco parejas (Carlos y Camila) era el hombre quien tenía mayor poder sobre las decisiones relacionadas con el dinero. Es importante señalar que tres de las cinco parejas que usaban este sistema, se encontraban por debajo del promedio del tiempo de convivencia de las parejas de primera unión $\mathrm{y}$, por lo tanto, podría decirse que se trataba de uniones relativamente jóvenes (5 años o menos).

Manejo independiente con fondo común. Este tipo de sistema es un híbrido entre los sistemas "manejo independiente" y "fondo común". En las parejas que adoptan este sistema, cada persona aporta de sus ingresos a una cuenta común que se utiliza para el sostenimiento del hogar y mantiene otra cuenta individual para sus gastos personales. Algunos autores han denominado este tipo de sistema "estrategia mixta" (Ashby \& Burgoyne, 2008). En esta muestra, tres parejas de primera unión tenían este sistema de administración del dinero (11,5\% de la muestra). Dos de estas parejas mencionaron que cada persona aportaba al fondo común en proporción a su salario, en tanto que 
en otra pareja (Andrés y Amalia) se dividían los gastos para aportar por igual al fondo común. Con respecto al control sobre el dinero, en dos parejas hombre y mujer compartían esta tarea; en otra pareja, el hombre tenía el control sobre las decisiones relacionadas con el dinero del fondo común.

Andrés (45 años, primera unión): "Nosotros simplemente hacemos una relación de gastos mensuales, llevamos un control de qué se gasta mensual y al final de mes, decimos: mire, eso se gastó ayer, tanto le corresponde a usted, tanto me corresponde a mí”.

\section{Percepción de justicia en la toma de decisiones}

El $95.8 \%$ de las parejas de primera unión y el $95.8 \%$ de las parejas reconstituidas coincidieron en que existe justicia en la forma como se administra y se toman decisiones sobre el dinero en el hogar. Es importante señalar que no sólo se trata de un porcentaje alto de participantes, sino que en él se encuentran representadas parejas que utilizan diferentes sistemas de administración del dinero. Tanto en parejas de primera unión como reconstituidas (10 parejas de cada tipo), el argumento principal para evaluar como justo el manejo del dinero en la relación de pareja fue que el sistema que tenían beneficiaba a todo el grupo familiar.

Enrique (31 años, primera unión): "Porque nos estamos beneficiando todos, no solamente yo... Lo vemos como una familia”.

Juliana (31 años, reconstituida): "Nosotros tomamos las decisiones bien, como lo más acertado para el hogar, ¿sí? Como la mejor decisión para que el hogar surja y vaya bien”.

Una segunda temática identificada en ambas muestras de parejas ( 8 parejas de primera unión y 5 reconstituidas) hace referencia a que es justa la forma como toman decisiones sobre el dinero porque se basa en acuerdos entre los miembros de la pareja.

Joaquín (33 años, primera unión): "Sí claro, sí es justo porque charlamos mucho, dialogamos mucho, o sea, me ha gustado siempre que ella me dé su opinión".

Sólo en algunos casos, uno de los miembros de la pareja percibía injusticia en la forma como se tomaban decisiones en su relación. Estos casos pertenecían a diferentes tipos de sistemas de manejo del dinero. Por ejemplo, Ignacio (primera unión, manejo independiente) manifestó que había injustica en la forma como se tomaban decisiones en su relación porque le gustaría que su pareja contribuyera más dinero al presupuesto familiar, y quisiera poder disponer de su dinero para gastos personales (ej., comprar una moto). Por su parte, Diana (salario completo), Eliana (mesada) y Miguel (fondo común), todos de parejas reconstituidas, mencionaron situaciones en las cuales el manejo del dinero fue injusto porque no se tuvieron en cuenta necesidades de los hijos.

\section{Discusión}

Este estudio se propuso indagar sobre los sistemas de administración del dinero que adoptan parejas de primera unión y reconstituidas. A continuación se analizan los resultados en relación con las preguntas planteadas en esta investigación y la literatura revisada sobre el tema.

\section{Aportes y administración del dinero}

Una de las preguntas de esta investigación se centró en los sistemas que implementan las parejas para administrar el dinero. El análisis realizado permitió identificar que en la muestra total los tres sistemas más utilizados eran el de fondo común, salario completo y mesada (cada uno representaba el 23\% de la muestra aproximadamente). A éstos seguía en frecuencia el sistema de manejo independiente (19\%). Estos resultados difieren de lo encontrado en otros estudios, donde se señala que el sistema de fondo común es preferido por la mayoría $(83 \%)$ de las parejas alrededor del mundo (Lauer \& Yodanis, 2011).

Al analizar los sistemas de administración del dinero por tipo de pareja (primera unión o reconstituida), también hubo resultados diferentes a lo que indican otros estudios. La literatura apunta que la ideología imperante con respecto al dinero en el primer matrimonio es compartir y, por lo tanto, se espera encontrar principalmente sistemas de fondo común (Burgoyne \& Morison, 1997). Sin embargo, en esta muestra de parejas de primera unión, ninguna empleaba el sistema de fondo común. Otros estudios han señalado que las parejas reconstituidas emplean principalmente el sistema de manejo independiente (Burgoyne \& Morison, 1997) o sistemas mixtos (manejo independiente con fondo común) (Pasley et al., 1994). En esta muestra 
no se encontraron parejas reconstituidas que emplearan estos sistemas.

Como se señaló en la introducción, existen factores que inciden en el tipo de sistema de administración del dinero que adoptan las parejas. Por una parte, algunos autores proponen que cuando los cónyuges contribuyen más equitativamente a los ingresos familiares, tienden a adoptar sistemas compartidos de administración del dinero (por ejemplo, sistema de fondo común) (Yodanis \& Lauer, 2007). Los resultados obtenidos en esta muestra no parecen apoyar esta hipótesis. En parejas de primera unión donde la discrepancia de salarios entre cónyuges era baja (igual o menor al 30\%), se encontraron sistemas de manejo independiente (3 parejas) y mixto (manejo independiente y fondo común) (1 pareja). De otro lado, sólo en dos de las parejas reconstituidas que empleaban el sistema de fondo común se observó una diferencia de ingreso menor al $30 \%$ entre los cónyuges. En las otras cuatro parejas que empleaban el sistema de fondo común, la discrepancia entre los ingresos de los cónyuges oscilaba entre 38 y $86 \%$. Esto parecería indicar que adoptar un sistema de fondo común no necesariamente se relaciona con una contribución equitativa en ingresos, como lo apuntan Yodanis y Lauer (2007).

Los estudios indican que cuando se percibe inestabilidad en la relación, las parejas prefieren sistemas de administración del dinero que les permitan separar fácilmente sus recursos en caso de una eventual disolución de la unión (Burgoyne \& Morison, 1997). Asimismo, Burgoyne, Clarke, Reibstein y Edmunds (2006) encontraron que las parejas recién casadas adoptan sistemas de manejo independiente o mixto, como una forma de mantener autonomía en la relación. Teniendo en cuenta que tres de las cinco parejas que empleaban el sistema de manejo independiente se encontraban en los primeros años del matrimonio (5 años o menos), se podría pensar que este factor influía en la manera como administraban el dinero. Empero, se requiere de otros estudios para evaluar esta hipótesis.

En relación con la estabilidad marital, otros autores han sostenido que las parejas en las cuales uno o ambos miembros tuvieron una relación anterior que terminó en divorcio, tienden a mantener el sistema de manejo independiente (Burgoyne \& Morison, 1997; Heimdal \& Houseknecht, 2003). Esta hipótesis no se corroboró en la muestra de parejas reconstituidas de este estudio. Por el contrario, estas parejas mostraron preferencia por el sistema de fondo común (43\%) y ninguna mantenía un sistema de manejo independiente.
Es necesario que futuras investigaciones sobre parejas de primera unión y reconstituidas en Colombia exploren específicamente los motivos que llevan a las parejas a adoptar diferentes sistemas de administración del dinero. Algunos estudios en otros países (Lauer $\&$ Yodanis, 2011) han planteado que las parejas que adoptan un sistema de fondo común tienen expectativas positivas sobre su relación y están satisfechas con su vida familiar. Entretanto, las parejas que adoptan un sistema de manejo independiente tienden a tener creencias individualistas sobre el matrimonio y mantienen una visión no tradicional sobre los roles de género. Estas y otras hipótesis requieren ser evaluadas empíricamente en Colombia.

\section{Control y toma de decisiones}

A pesar de que en aproximadamente el $60 \%$ de las parejas existían diferencias mayores al 50\% en el salario de los cónyuges, es interesante resaltar que esta situación no necesariamente llevaba a señalar a quien tenía más ingresos como proveedor principal. Esto sólo ocurría cuando a una sola persona sostenía económicamente a la familia (parejas con sistema de mesada). En general, las parejas señalaban que los dos aportaban por igual $(3,8 \%)$ o proporcionalmente a sus ingresos $(61,5 \%)$ para cubrir los gastos del hogar. Esta percepción sobre quién sostenía económicamente la familia, podría explicar por qué un porcentaje alto de la muestra (69\%) reportó que el control del dinero también era compartido por los dos cónyuges. Este porcentaje es superior al encontrado en otros estudios en Colombia, donde el 50\% de las mujeres reportan tomar decisiones con su pareja sobre gastos del hogar (Profamilia, 2010). No obstante, contrario a los resultados de dicho estudio, donde las mujeres que aportaban menos a los ingresos familiares tenían menor control de las decisiones sobre el dinero (Profamilia, 2010), las parejas de este estudio que empleaban sistemas de salario completo o de mesada (12 parejas) mantenían en su mayoría (58\%) un control compartido de las decisiones sobre los ingresos.

A diferencia de lo hallado en otros estudios (Yodanis \& Lauer, 2007) sobre el mayor control ejercido por el hombre en parejas de segunda unión, en la mayoría de las parejas reconstituidas que empleaban un sistema de fondo común los dos miembros de la pareja tenían control conjunto sobre decisiones relacionadas con el dinero. 


\section{¿Sistemas justos?}

La percepción de justicia en la toma de decisiones sobre el dinero parece ser independiente del sistema de administración empleado por las parejas en esta muestra. Tanto parejas que empleaban sistemas de salario completo y mesada, como aquellas que manejaban un fondo común percibían justicia en las decisiones. Las parejas percibían sus decisiones como justas porque beneficiaban a todos. Esta concepción se ha denominado justicia basada en necesidades (Jacobson, 1993) y contrasta con otras concepciones de justicia en la distribución de los ingresos identificadas en las relaciones de pareja (equidad e igualdad).

Por otra parte, los participantes señalaron que las decisiones eran justas porque se basaban en acuerdos entre los cónyuges. Otros estudios también han señalado que la percepción de justicia aumenta cuando ambos cónyuges participan expresando sus puntos de vista (justicia procedimental) (Kluwer, Heesink \& van de Vliert, 2002).

\section{Limitaciones del estudio e investigaciones futuras}

Esta investigación se basó en un estudio de carácter exploratorio sobre las experiencias de parejas en familias de primera unión y reconstituidas con respecto a la administración del dinero. Por lo tanto, se requiere de otros estudios en el futuro que superen las limitaciones discutidas a continuación. Una de las limitaciones de las conclusiones de este estudio tiene que ver con las características de la muestra. Se trataba principalmente de una muestra intencional conformada por hombres y mujeres residentes en Bogotá, con educación secundaria o educación universitaria, y un tiempo promedio de convivencia entre los 6 (parejas reconstituidas) y los 13 años (parejas primera unión). Es necesario entonces que en futuras investigaciones se utilicen métodos probabilísticos de muestreo, que permitan obtener mayor variabilidad en las características demográficas de los participantes. Esto permitiría identificar variaciones en los sistemas de administración empleados por parejas que habitan en contextos rurales y urbanos, con diferentes niveles educativos, y en diferentes etapas de la relación de pareja y formación de la familia.

Por otra parte, esta investigación se centró en el análisis de entrevistas en profundidad. A pesar de que las entrevistas han sido empleadas en otros estudios sobre el tema (Burgoyne et al., 2006), sería útil adoptar una combinación de estrategias (cuestionarios y entrevistas) para explorar con mayor detalle asuntos como los siguientes: a) ¿qué criterios emplean las parejas en las decisiones sobre distribución y manejo de los ingresos (equidad, igualdad o necesidad)?; b) ¿quién ejerce mayor control en decisiones sobre aspectos específicos del presupuesto (gastos diarios vs. inversiones a largo plazo)?; c) ¿qué tanta autonomía tienen los miembros de la pareja en decisiones sobre dinero destinado a gastos personales? ¿Difiere el grado de autonomía de acuerdo con el sexo, nivel de ingresos y el aporte de cada persona al presupuesto de gastos?; y d) ¿qué factores se encuentran asociados a la percepción de justicia en las decisiones sobre la administración del dinero: valores y expectativas, grupo de referencia (Kluwer et al., 2002) o procedimientos empleados en la toma de decisiones? ¿Varían esos factores de acuerdo con el sistema de administración empleado (por ejemplo, fondo común, manejo independiente)?

De igual manera, investigaciones futuras deberían incorporar variables tanto individuales como del contexto mencionadas en la introducción de este manuscrito, con el propósito de evaluar su efecto diferencial en la implementación de los diferentes sistemas de administración identificados en este estudio en parejas de primera unión y reconstituidas. En particular, sería interesante evaluar el efecto de creencias y expectativas en relación con roles de género, e ideologías sociales sobre la institución matrimonial y la concepción institucional del proveedor.

Por último, es necesario que la investigación empírica sobre los sistemas de administración del dinero y la toma de decisiones sobre asuntos económicos sea la base en el diseño de intervenciones clínicas con parejas tanto de tipo preventivo como terapéutico. En el campo de la terapia de pareja (Guerin, Fay, Burden \& Kautto, 1987) y en la investigación sobre dinámicas de la relación de pareja (Anderson \& Sabatelli, 2007) se ha reconocido que la administración del dinero es una de las áreas de funcionamiento del matrimonio que se relaciona directamente con la distribución del poder. Específicamente, se ha señalado que quien aporta más recursos económicos posee por lo general más poder en las decisiones sobre cómo debe invertirse el dinero y que dicho poder se reconoce como legítimo, especialmente cuando existen acuerdos explícitos o implícitos entre los miembros de la pareja (Anderson \& Sabatelli, 2007). Sin embargo, puede ocurrir que quien aporte más recursos intente utilizar dicho poder para tomar control absoluto sobre decisiones económicas y de otra índole $\mathrm{y}$, de esta manera, se rompan acuerdos establecidos por la pareja y se presenten conflictos (Guerin et al., 1987). 
En tales casos, el terapeuta interviene para restablecer el equilibrio de poder en la relación, de manera que ambos miembros de la pareja perciban equidad y justicia.

Los resultados de este estudio nos invitan a considerar varios aspectos en el diseño de intervenciones tanto preventivas como terapéuticas dirigidas a parejas. En primer lugar, los terapeutas deben incluir en sus protocolos de evaluación no sólo preguntas acerca del sistema que emplean las parejas para administrar el dinero (mesada, fondo común u otros), sino también evaluar en detalle los acuerdos establecidos para controlar el dinero. En las parejas de este estudio se encontró que aunque dos parejas tengan el mismo sistema de administración (por ejemplo, fondo común), sus acuerdos sobre quién tiene control sobre decisiones relacionadas con el dinero pueden diferir. En este sentido, al evaluar las dinámicas de relación alrededor del dinero es necesario tener en cuenta la percepción de cada individuo sobre el grado de equidad y justicia tanto en el sistema de administración como en el control del dinero como tal. Esto, a su vez, determinará los objetivos de la intervención que se diseñe para restaurar el equilibrio en la relación. En el caso de parejas reconstituidas, la evaluación debe considerar los acuerdos establecidos por la pareja acerca de aspectos como la administración de sus bienes y del dinero para gastos relacionados con los hijos de uniones anteriores. En segundo lugar, en el diseño de intervenciones dirigidas a parejas debe tomarse en cuenta si las dificultades en el manejo del dinero se originan en el sistema adoptado por la pareja o en diferencias en la filosofía de cada uno acerca del dinero. Como lo señalan varios autores (Guerin et al., 1987; Jacobson \& Christensen, 1996), uno de los miembros de la pareja puede ser más liberal en el manejo del dinero (gasta excesivamente), mientras que el otro puede ser más conservador y demostrar más preocupación por cómo se gasta y administra el dinero. Estas diferencias de pensamiento sobre el valor del dinero y su uso deben evaluarse cuidadosamente e intervenir para lograr aceptación y tolerancia hacia el punto de vista del cónyuge (Jacobson \& Christensen, 1996). Por último, las intervenciones preventivas diseñadas para parejas que se preparan para casarse, o recién casadas, generalmente contemplan entre sus objetivos el desarrollo de habilidades de comunicación dirigidas a mejorar la toma de decisiones sobre diferentes aspectos de la relación, incluyendo el manejo del dinero (Stahmann \& Hiebert, 1997). Tanto en parejas de primera unión como en parejas reconstituidas, un aspecto central en estas intervenciones debería ser el diálogo sobre expectativas acerca de las decisiones sobre el dinero y la manera como cada persona espera que se administren los recursos en la relación. Como se mencionó en la introducción, especialmente las mujeres que vuelven a casarse esperan mayor equidad en la toma de decisiones (Ganong \& Coleman, 2004). Estas expectativas sobre lo que cada individuo considera una participación equitativa en las decisiones deben aclararse en el proceso de preparación para una nueva unión marital puesto que, posiblemente, esto será de utilidad en la prevención de dificultades en el futuro. A medida que las parejas adoptan modelos basados en la distribución equitativa de responsabilidades y decisiones, los objetivos de los programas de intervención deberían revisarse y adecuarse a las experiencias de las parejas en nuestro tiempo. En este sentido, se requiere continuar investigando sobre las expectativas sobre manejo del dinero, así como sobre procesos de negociación que desarrollan las parejas en este tema.

A manera de conclusión, es posible afirmar que los sistemas de administración del dinero implementados por parejas de primera unión y reconstituidas son diversos. Existen diferencias entre los hallazgos de estudios realizados en otros países y esta investigación, que deberían motivar futuros estudios en Colombia y otros países latinoamericanos sobre los factores que se asocian a las decisiones de las parejas sobre el dinero. Estudios indican que los conflictos de pareja alrededor de la administración del dinero tienden a ser recurrentes, generalmente son de difícil solución, y tienen implicaciones tanto a corto como a largo plazo (Papp, Cummings \& Goeke-Morey, 2009). Por lo tanto, se requieren estudios que ayuden a establecer no sólo las estrategias que adoptan las parejas, sino también los procesos de negociación sobre el manejo del dinero que ocurren en las relaciones. Por último, como se explicó anteriormente, esta línea de investigación puede ser de gran utilidad en el diseño de programas de intervención tanto para parejas de primera unión como reconstituidas.

\section{Referencias}

Alvis-Guzmán, N., Yánez-Contreras, M., Quejada-Pérez, R., Acevedo-González, K., \& Del Río-Carrasquilla, F. (2010). Fecundidad y participación de la mujer en el mercado laboral en la Costa Caribe y en Colombia. Revista Gerencia y Políticas de Salud, 9(19), 90-107.

Amato, P., Booth, A., Johnson, D., \& Rogers, S. (2007). Alone together: How marriage in America is changing. Cambridge, MA: Harvard University Press.

Anderson, S., \& Sabatelli, R. (2007). Family Interaction: A multigenerational developmental perspective ( $4^{\mathrm{a}}$. ed.). Boston: Allyn \& Bacon. 
Blood, R., \& Wolfe, D. (1960). Husbands and wives: The dynamics of married living. New York: Free Press.

Blumstein, P., \& Schwartz, P. (1985). American couples: Money, work, and sex. New York: Pocket Books.

Blumstein, P., \& Schwartz, P. (1991). Money and ideology: Their impact on power and the division of household labor. En R. Blumberg (Ed.), Gender, family, and economy: The triple overlap (pp. 261 - 288). Newbury Park, CA: Sage.

Brinton, M. \& Nee, V. (Eds.). (1998). The new institutionalism in sociology. New York: Russell Sage.

Burgoyne, C. (1990). Money in marriage: How patterns of allocation both reflect and conceal power. The Sociological Review, 38(4), 634-665.

Burgoyne, C. (2004). Heart-strings and purse-strings: Money in heterosexual marriage, Feminism and Psychology, 14(1), 165-172.

Burgoyne, C., Clarke, V., Reibstein, J., \& Edmunds, A. (2006). All my godos I share with you? Managing Money at the transition to heterosexual marriage. The Sociological Review, 54(4), 619-637.

Burgoyne, C. \& Lewis, A. (1994). Distributive justice in marriage: equality or equity? Journal of Community and Applied Social Psychology, 4(2), 101-114.

Burgoyne, C. \& Morison, V. (1997). Money in remarriage: Keeping things simple and separate. Sociological Review, 45, 363-395.

Burgoyne, C. Reibstein, J., Edmunds, A., \& Dolman, V. (2007). Money management systems in early marriage: Factors influencing change and stability. Journal of Economic Psychology, 28, $214-228$.

Burgoyne, C. Reibstein, J., Edmunds, A., \& Routh, D. A. (2010). Marital commitment, money and marriage preparation. What changes after the wedding? Journal of Community and Applied Social Psychology, 20, 390 - 403.

Burgoyne, C. \& Routh, D. (2001). Beliefs about financial organization in marriage: The 'Equality Rules OK' norm? Zeitschrift fuer Sozialpsychologie, 32, 162-170.

Cherlin, A. (2004). The deinstitutionalization of American marriage. Journal of Marriage and Family, 66, 848-861.

Coleman, M., \& Ganong, L. (1989). Financial management in stepfamilies. Lifestyles: Family and Economic Issues, 10, 217-232.

Coontz, S. (2005). Marriage, a history: From obedience to intimacy or how love conquered marriage. New York: Viking.

Fishman, B. (1983). The economic behavior of stepfamilies. Family Relations, 32, 359-366.

Ganong, L. \& Coleman, M. (2004). Stepfamily relationships: Development, dynamics, and interventions. New York: Kluwer Academic.

Galvis, L. (2010). Diferenciales salariales por género y región en Colombia: Una aproximación con regresión por cuartiles. Revista de Economía del Rosario, 13(2), 235-277.

Guerin, P., Fay, L. Burden, S. \& Kautto, J. (1987). The evaluation and treatment of marital conflict. New York: Basic Books.

Heimdal, K. \& Houseknecht, S. (2003).Cohabiting and married couples' income organization: Approaches in Sweden and the United States. Journal of Marriage and Family, 65, 525 - 538.

Jacobson, D. (1993). What's fair? Concepts of financial management in stepfamily households. Journal of Divorce and Remarriage, 19(3), 221-238.

Jacobson, N., \& Christensen, A. (1996). Acceptance and change in couple therapy. New York: Norton.
Kluwer, E. Heesink, J. \& Van de Vliert, E. (2002). The division of labor across the transition to parenthood: A justice perspective. Journal of Marriage and Family, 64, 930-943.

Lauer, S. \& Yodanis, C. (2011). Individualized marriage and the integration of resources. Journal of Marriage and Family, 73, 669-683.

Martin, P. (2004). Gender as social institution. Social Forces, 82, $1249-1273$.

Mintz, S., \& Kellogg, S. (1988). Domestic revolutions: A social history of American family. New York: Free Press.

Muhr, T. (2004). ATLAS.ti 5.0 User's manual (2a ed.). Berlin: Scientific Software Development.

Oropesa, R. Landale, N., \& Kenkre, T. (2003). Income allocation in marital and cohabiting unions. Journal of Marriage and Family, $65,910-926$.

Pahl, J. (1989). Money and marriage. Basingstoke, U.K.: Macmillan.

Papp, L. Cummings, E. \& Goeke-Morey, M. C. (2009). For richer, for poorer: Money as a topic of marital conflict in the home. Family Relations, 58, 91-103.

Pasley, K., Sandras, E., \& Edmondson, M. (1994). The effects of financial management strategies on the quality of family life in remarriage. Journal of Family and Economic Issues, 15, 53-70.

Profamilia. (2010). Encuesta Nacional de Demografía y Salud ENDS 2010. Recuperado de http://encuestaprofamilia.com

Stahmann, R. \& Hiebert, W. (1997). Premarital and remarital counseling: The professional's handbook. San Francisco: Jossey-Bass.

Strauss, A. (1987). Qualitative analysis for social scientists. Cambridge: Cambridge University Press.

Strauss, A. \& Corbin, J. (1990). Basics of qualitative research: Grounded theory procedures and techniques. London: Sage.

Tichenor, A. (1999). Status and income as gendered resources: The case of marital power. Journal of Marriage and the Family, 61, $638-650$.

Treas, J. (1993). Money in the bank: Transaction costs and the economic organization of marriage. American Sociological Review, $58,723-734$.

Vogler, C. (2005). Cohabiting couples: Rethinking money in the household at the beginning of the twenty first century. Sociological Review, 53, 1- 29.

Vogler, C., Brockmann, M., \& Wiggins, R. (2006). Intimate relationships and changing patterns of money management at the beginning of the twenty first century. British Journal of Sociology, 57, $455-482$.

Vogler, C., \& Pahl, J. (1993). Social and economic change and the organization of money within marriage. Work, Employment, \& Society, 7, $71-95$.

Webley, P., Burgoyne, C., Lea, S. \& Young, B. (2001). The Economic Psychology of Everyday Life. East Sussex, UK: Psychology Press.

Willig, C. (2001). Introducing qualitative research in Psychology: Adventures in theory and method. Berkshire: Open University Press.

Wilson, G. (1987). Money in the Family. Avebury: Aldershot.

Yodanis, C., \& Lauer, S. (2007). Managing money in marriage: Multilevel and cross-national effects of the breadwinner roles. Journal of Marriage and Family, 69, 1307-1325.

Zelizer, V. (1989). The social meaning of money: "Special monies." American Journal of Sociology, 95, 342 - 377. 
